Management model of care: the innovation of nursing to ensure the welIbeing of patients at the General Hospital "Dr. Manuel Gea González" on the Mexico city

\title{
Modelo de gestión del cuidado: innovación de enfermería para garantizar el bienestar de los pacientes, en el Hospital General "Dr. Manuel Gea González", en la Cd. de México.
}

Mtra. Wendy Melina Suasto Gómez, ${ }^{1}$ Dra. Lasty Balseiro Almario, ${ }^{2}$ Dra. Irma Jiménez Escobar, ${ }^{3}$ Mtra. Ana Lilia Hernández Hernández, ${ }^{4}$ Mtra. Ma. Adriana Chaparro Sánchez, ${ }^{5}$

Pste. Jessica Paulina Adán Ruíz ${ }^{6}$

\begin{abstract}
Nursing healthcare management implies using professional judgement regarding the issues of planning, organization, integration, direction, and control, in order to provide timely, safe, and integral healthcare and achieve and sustain the wellbeing of persons. This human process implies the continuous interaction between healthcare professionals and persons, sick or healthy. To provide this care, nursing professionals manage interdisciplinary interactions with other healthcare professionals in a process which often involves human, physical, material, and financial resources. To address these complex issues, the new Nursing Sub-Direction Management 2017-2022 has designed a Nursing Healthcare Management Model which highlights administration innovations which allow the graphical representation of the situations acknowledging the important tasks which the nursing personnel carry out at "Dr. Manuel Gea González" General Hospital to guarantee a healthcare of quality. This Management Model has its theoretical and methodological foundations on four dimensions: Administration of Health Services; Health; Care; and Persons. Under this model, nursing personnel can develop their main functions of teaching, management, and research, and enhance the interaction and coordination with healthcare interprofessional teams.
\end{abstract}

Keywords: wellbeing, nursing, Healthcare Management, model, persons.

Citación: Suasto-Gómez W. M., Balseiro-Almario L, Jiménez-Escobar I, Hernández-Hernández A. L., Chaparro Sánchez M. A., Adán-Ruíz J. P. Modelo de gestión del cuidado: innovación de enfermería para garantizar el bienestar de los pacientes, en el hospital general "Dr. manuel Gea González", en la Cd. de México. Rev Enfer Neurol 2018;17(3):pp 39-48.

* Correspondencia: Wendy Melina Suasto Gómez. Email: wendy_suasto@hotmail.com

${ }^{1}$ Subdirectora de Enfermería del Hospital General Dr. Manuel Gea González.

${ }^{2}$ Profesora de Carrera Titular "B" de la ENEOUNAM.

${ }^{3}$ Directora Médica del Hospital General Dr. Manuel Gea González.

${ }^{4}$ Jefe del Área Clínica de Enfermería del Hospital General Dr. Manuel Gea González.
${ }^{5}$ Jefe del Departamento de Docencia e Investigación de Enfermería del Hospital General ${ }^{6}$ Dr. Manuel Gea González. Pasante de la Lic. en Enfermería y Obstetricia de la ENEO- UNAM.

Recibido: 10 de julio 2018

Aceptado: 30 de agosto 2018 


\section{Resumen}

La gestión del cuidado de enfermería implica la aplicación de juicios profesionales en la planeación, organización, integración, dirección y control para brindar con la planeación estratégica, cuidados oportunos, seguros e integrales que garanticen la continuidad de la atención para obtener el bienestar y la salud de las personas. Este bienestar se logra mediante el proceso humano y social de alteridad entre un sujeto que es el profesional de enfermería y otro sujeto, que es la persona sana o enferma con algún quebrante de salud. Para proporcionar el cuidado, los profesionales de enfermería realizan gestiones de interacción interdisciplinaria con otros profesionales de la salud y adaptaciones de factores humanos y recursos físicos, materiales y financieros que buscan garantizar la continuidad día a día de la calidad de los cuidados. En esta nueva gestión de la Subdirección de Enfermería (20172022) consciente de la nueva responsabilidad y el compromiso institucional del cuidado, se ha creado un Modelo de gestión del cuidado de Enfermería, en el que se plasma una innovación administrativa que permite mediante una representación gráfica (Modelo de gestión) evidenciar el importante trabajo que realiza el personal de Enfermería del Hospital General "Dr. Manuel Gea González", para garantizar la calidad del cuidado. El Modelo de Gestión sienta sus bases teórico-metodológicas en 4 dimensiones: administración de los servicios, salud, cuidado y persona. Específicamente, la enfermería desarrolla también en este modelo, sus principales funciones: servicios, docencia, administración e investigación, lo que le permite interactuar y coordinarse con el equipo interdisciplinario de salud para proporcionar cuidados integrales para individuos sanos y enfermos.

Palabras clave: bienestar, enfermería, gestión del cuidado, modelo, personas.

\section{Introducción}

El presente ensayo, tiene por objeto dar a conocer el Modelo de gestión del cuidado como una innovación de enfermería para garantizar el bienestar de los pacientes en el Hospital General "Dr. Manuel Gea González”, de la Cd. de México. Por ello, siguiendo el desarrollo secuencial de éste, se presentan a continuación los conceptos básicos de modelos de gestión del cuidado de enfermería, el planteamiento de la tesis, argumentación teórica, conclusiones y referencias.
Concepto de modelo: para Carvajal A. ${ }^{1}$ el significado etimológico de la palabra "Modelo" implica a. medida, cantidad, proporción, b. ejemplar o prototipo, y c. un algo proporcionando a otra cosa. Sin embargo, para Wartofsky M. ${ }^{2}$ un modelo es una versión de representación de algo tomado del original. Es decir, que la nueva entidad se produce al imitar al original. Lo anterior da idea de que un Modelo resulta ser la representación gráfica de una realidad práctica o bien, una espe- 
cie de descripción o representación de la realidad que pueden ser: hechos, situaciones, fenómenos, estructuras y sistemas, entre otros, que por lo general, está en función de supuestos teóricos.

Modelos de gestión: para Martínez A. ${ }^{3}$ el principal objetivo de crear modelos de gestión en las empresas y organizaciones es para ofrecer productos o servicios que permitan satisfacer las demandas de los usuarios en un entorno competitivo. En este sentido, no solamente hay que hacer las cosas bien, sino hacerlas mejor que los demás; es decir, innovar con estrategias directivas que garanticen los servicios.

Por otro lado, la palabra gestión que viene del latín gestio, que hace referencia según Pérez J. y Merino M. ${ }^{4}$ a la acción y a la consecuencia de hacer diligencias para lograr algo. Estas diligencias permiten y hacen posible la realización de una operación comercial o de cualquier anhelo. Entonces, la noción de la gestión, se entiende hacia el conjunto de trámites que se llevan a cabo para lograr un objetivo. Por ello, para lograr los modelos de gestión se requiere de la planeación estratégica y la gestión de factores humanos y recursos físicos, materiales y financieros para alcanzar las metas y los objetivos de la organización y de sus clientes. Por tanto, se hace necesario conocer la filosofía organizacional con la formulación clara de la misión, visión, valores y estrategias de acción.

Modelos de gestión de enfermería: la creación de los modelos de gestión de enfermería nacen según Kerouac, et al., ${ }^{5}$ del papel que desempeña la enfermera gestora al planificar, organizar, dirigir y controlar los factores humanos y los recursos físicos, materiales y financieros con la intención de atender, con la mejor eficacia posible, los objetivos de la organización. Así, la enfermera gestora guía su actividad a partir de los conocimientos extraídos de las ciencias de la orga- nización, gestión, economía, sociología y política, entre otras, para brindar ayuda a las personas.

Entonces, al ser la gestión de los cuidados un proceso heurístico dirigido a movilizar factores humanos, recursos de distinta índole y del entorno para favorecer el cuidado a las personas, que interaccionan con su ambiente y viven experiencias de salud. Por ello, la enfermera gestora requiere creatividad, organización, liderazgo, delegación y control de las acciones del personal cuidador, para contribuir con el bienestar de las personas que se cuidan. Así, para realizar las acciones de cuidado, la gestora de enfermería se relaciona día a día con múltiples equipos interdisciplinarios en donde priva la colaboración y el compromiso para con los individuos sanos o enfermos. En este proceso de colaboración, es la enfermera gestora quien coordina las acciones encaminadas al bienestar de los pacientes que se traducen en apoyo, ayuda y servicio. Esa es la esencia del cuidado.

\section{Planteamiento de la tesis}

\section{Modelos de gestión del cuidado de las teóri-} cas de enfermería. Para Morfi R. ${ }^{5}$ la gestión del cuidado de enfermería, se define como la aplicación de un juicio profesional en la planificación, organización, motivación y control de la provisión de cuidados, óptimos, seguros e integrales, que aseguren la continuidad de la atención y que se sustenten en lineamientos estratégicos, para obtener como producto final, la salud y que ésta se vincule directamente con el bienestar de los individuos y las poblaciones.

Por lo anterior, la gestión de los cuidados tiene el propósito de adecuar la atención y los cuidados a las necesidades de las personas sanas y enfermas y a la demanda de sus familiares, cuida- 
dores y comunidad en general. $Y$ en este proceso de cuidar, emergen los modelos de gestión del cuidado de las teóricas de enfermería: Henderson, Orem, King, Roy, Royers, Leininger, entre otros, que como plantea Kerouac S. et al., ${ }^{6}$ guían las actividades de dirección de los cuidados hacia el bienestar de las personas. Es decir, la concepción de la disciplina de enfermería al realizar la práctica clínica, debe ir acompañada de una práctica administrativa efectiva. Es allí, donde las teóricas de enfermería proponen nuevos caminos para realizar la gestión guiada por el cuidado de las personas, agrupadas en seis escuelas: las necesidades, interacción, efectos deseables, promoción de salud, el ser humano unitario y el caring, entre otras, que a continuación se presentan:

Dorothea E. Orem: para Raile M, et al., ${ }^{7}$ Orem plantea la teoría del déficit del autocuidado que se refiere a las acciones que realiza una persona con el fin de promover y mantener su salud. Para ella las enfermeras gestoras deben tener no solamente un conocimiento renovador de los cuidados de enfermería, sino llevar éstos a la práctica. Para Berbiglia V.A y Banfield B. ${ }^{8}$ el autocuidado de Orem se refiere a las acciones que realiza una persona con el fin de preservar y mantener la vida, salud y bienestar. Así, la concepción de Orem guía al personal de enfermería al utilizar el enfoque centrado en las necesidades de salud de las personas que tengan dificultades o incapacidades con respecto a su autocuidado.

Imogene King: teórica de la escuela de las "interacciones", considera que promover la salud presupone acciones, reacciones, interacciones y transacciones que tienen lugar en 3 entornos: personal, interpersonal y social. Así, la gestora de enfermería se mueve en estos 3 entornos e inte- racciona con ellos para satisfacer las necesidades de las personas. Para Sieloff C.L y Messmer P.R ${ }^{9}$ King conceptualiza a la enfermería como un proceso de acción, reacción e interacción en el que la enfermera y el cliente comparten información sobre sus percepciones. Esto implica que la gestora de enfermería promueve la salud en los entornos donde se producen los cuidados, porque las organizaciones de salud constituyen una parte importante del entorno de cada persona. Por ello, King utiliza un marco de sistemas y subsistemas de la organización hospitalaria como un todo.

Callista Roy: teórica de la escuela de los "efectos deseables" propone un modelo para la práctica de la administración basada en la teoría de la adaptación y de los sistemas. Así, para Roy la supervivencia y el crecimiento de la organización están agrupados por la capacidad de adaptación de las instituciones y los estímulos internos y externos generadores del cambio. Entonces, para cuidar, la enfermera gestora recurre a diversos mecanismos estabilizadores e innovadores para lograr el equilibrio y el cambio. Por ello, para Phillips K. D y Harris R. ${ }^{10}$ Roy propone sistemas de adaptaciones con entradas y salidas de información con procesos de atención y de retroalimentación. Para Roy la gestión de los cuidados consiste en asegurar la práctica de los servicios de calidad a los clientes. Así, los supuestos surgidos de las teorías de sistemas y de la teoría de adaptación son la base del modelo de gestión de Roy.

Martha Royers: se sitúa en la escuela del "ser humano unitario". La idea principal de Royers es la interacción entre los campos "persona" y "entorno". Entonces, la gestión ocurre en estos dos campos con la organización como respaldo. Así, la enfermera gestora conociendo los componen- 
tes de la organización realiza la interacción con la práctica administrativa para promover los cuidados considerando siempre a la persona como un ser integral y unitario. De igual forma, para Gunter M. E. ${ }^{11}$ la ciencia de Royers ve al hombre como un todo, con consecuencias importantes en la gestión del cuidado en donde la enfermería traducida en ciencia y arte, se centra en el interés por las personas y por el mundo en que viven éstas personas, integrándolas con su entorno, por fomentar la salud y el bienestar.

Madeleine Leininger: de la escuela del "caring", el cuidado transcultural en donde la enfermera gestora tiene en cuenta elementos como la cultura y la universalidad para brindar el cuidado. Por ello, la enfermera gestora deberá entonces, ejercer un liderazgo proactivo desde los valores, las creencias y la cultura de las personas para brindar los cuidados y el bienestar. De igual forma, la filosofía del "caring" es de un valor humanista, que la gestora de enfermería toma en cuenta al brindar los cuidados. Así, para McFarland M. R. ${ }^{12}$ la teoría de Leinninger se basa en las disciplinas de la antropología y de la enfermería. De hecho, la enfermería transcultural es el área principal que debe tener en cuenta la gestora de enfermería y que se centra en el estudio comparativo y el análisis de las diferentes culturas y subculturas del mundo con respecto a los valores sobre cuidados, de experiencia y carencias de salud y enfermedad.

Modelo de gestión del cuidado de enfermería del Hospital Gea González: según Suasto W. M. ${ }^{13}$ la enfermería del Hospital General “Dr. Manuel Gea González" tiene en esta nueva gestión (2017- 2022) claramente definida una filosofía renovadora hacia el cuidado con la promulgación de la: misión, visión, valores y estrategias de enfermería que buscan aumentar los resultados óptimos del cuidado a todos los niveles de la organización hospitalaria, para brindar bienestar a las personas y sus familias (ver figura 1: Modelo de gestión del cuidado de enfermería del Hospital General “Dr. Manuel Gea González”). 
Figura 1. Modelo de gestión del cuidado de enfermería del Hospital General“Dr. Manuel Gea González”

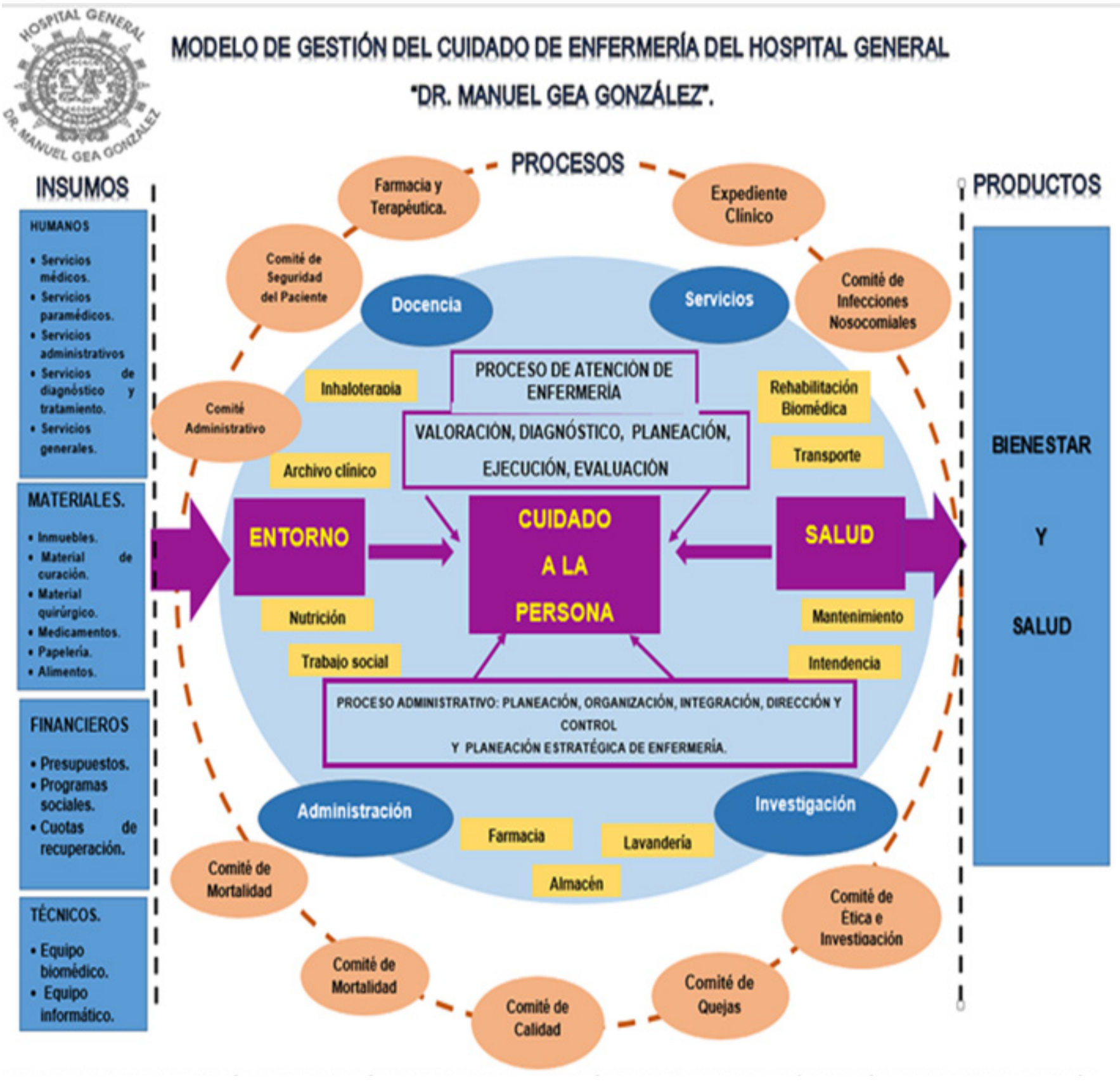

Fuente: Suasto WM. Modelo de gestión del cuidado de enfermería del Hospital General "Dr. Manuel Gea González". Seminario permanente de reingeniería en enfermería. Hospital General “Dr. Manuel Gea González”. Ciudad de México. Agosto, 2018. p.1. 
Por lo anterior, los sustentos teóricos que fundamentan el Modelo de gestión de la enfermería, en particular tiene sus bases en 4 dimensiones: administración, salud, enfermería y cuidado a las personas, como a continuación se explican:

La dimensión de administración: según Balseiro L. ${ }^{14}$ es la ciencia, técnica y arte, que según diversos criterios, permite coordinar los factores humanos y recursos físicos, materiales y financieros para el logro de los objetivos. Los teóricos Frederick Taylor y Henry Fayol reconocieron desde la Revolución Industrial, los principios básicos de la administración eficaz. Más tarde, según Guillen D.A. ${ }^{15}$ Elton Mayo, Max Weber, Douglas McGregor, Rensis Likert y otros, redefinieron los principios básicos y los transformaron en el conocimiento administrativo operante actual. Así, la administración, permite integrar factores y recursos que la gestión de enfermería administra, tomando las mejores decisiones para el bienestar de las personas.

Por lo anterior, el Modelo de gestión del cuidado de enfermería creado por la presente dirección del Hospital General "Dr. Manuel Gea González" tiene como propósito aplicar las teorías del proceso administrativo y planeación estratégica en los factores y recursos, para adecuar los cuidados a las necesidades de los enfermos y a las demandas de las familias. Estas acciones permitirán también emplear el conocimiento administrativo para poder movilizar los recursos y producir bienestar en las personas. Además, el Modelo de gestión del cuidado también tiene los fundamentos de la teoría general de los sistemas, que para Domínguez V.A. y López M. A. ${ }^{16}$ fue creada por Ludwig Von Bertalanffy, aunque años más tarde, Oscar Johansen Bertoglio le imprime el enfoque interdisciplinario. Por ello, Tamayo A. ${ }^{17}$ considera que la metodología de esta teoría permite visualizar a la organización como un todo, tomando como base sus componentes de interrelación e interacción de todos los sistemas y subsistemas.

La dimensión de salud. el Modelo de gestión del Hospital General "Dr. Manuel Gea González" tiene sus fundamentos teóricos en el modelo de calidad de Avedis Donabedian, quién según Torres M. ${ }^{18}$ establece las dimensiones de estructura, proceso y resultados, con indicadores que permiten medir la calidad en salud. Así, en la estructura se miden la cantidad y calidad del personal, equipos e instrumentos, recursos financieros, instalaciones, físicas, normas, reglamentos, procedimientos y sistemas de información. En el proceso, las acciones del personal y de los pacientes; en la comunicación la aplicación de los procedimientos, entre otras; y en resultados el cumplimiento de los indicadores, gastos efectuados, mejoramiento de la salud del paciente, satisfacción de los usuarios y la acreditación institucional, etcétera.

Para Donabedian A. ${ }^{19}$ la atención a la salud tiene tres componentes: atención técnica, manejo de la relación interpersonal y el ambiente en el que se lleva a cabo el proceso de atención. Por lo tanto, la evaluación de cada uno de estos aspectos permite estimar la calidad de la atención brindada. En consecuencia, el Modelo de gestión del cuidado de enfermería del Hospital General Dr. Manuel Gea González retoma las dimensiones de estructura, proceso y resultado de Donabedian para que la enfermera gestora pueda movilizar los factores y recursos, en las áreas de bienestar en las personas. Aquí la idea principal es buscar siempre la calidad de los cuidados

La dimensión de enfermería: ésta aporta la gestión y el cuidado. Con la gestión se realizan todas las diligencias, acciones, operaciones e intervenciones de coordinación por turnos para la 
atención de calidad a las personas. Para el cuidado, se toma en cuenta el metaparadigma: entorno, salud, cuidado y persona, que para Durán M. ${ }^{20}$ son declaraciones que identifican los fenómenos relevantes a la disciplina. Por ejemplo, el entorno es el contexto de la persona y el ambiente con el que coexiste. La salud es la meta de enfermería hacia el enfermo y el sano. Los cuidados son las intervenciones de las enfermeras para las personas, y estas últimas son un todo indivisible con componentes: biológicos, psíquicos, espirituales y sociales. Además, en su actuar profesional la enfermera gestora se auxilia de la metodología del proceso de atención de enfermería (PAE) que para González P, et al., ${ }^{21}$ consiste en una serie de fases: valoración, diagnóstico de enfermería, planeación de actividades, ejecución y evaluación, que permite de forma ordenada y sistemática, brindar los cuidados, a través del enfoque individualizado de la solución de problemas. Esta metodología científica llamada PAE que data de los 80’s implica según Garbey J. y Cols ${ }^{(22)}$ un proceso de conocimiento resultante de la interacción ente el sujeto y el objeto del conocimiento para brindar cuidados específicos e individualizados. Hay por ello, una similitud entre el método científico y el proceso de atención de enfermería, lo que ha permitido realizar los cuidados con una base científica, al tomar en cuenta las teorías y leyes de las diferentes ciencias naturales.

De la misma manera, la enfermería aporta de manera proactiva el desarrollo de sus principales funciones: servicios, docencia, administración e investigación para atender y apoyar a los individuos sanos y enfermos. Estos servicios que son únicos en los profesionales de enfermería, interactúan con los servicios hospitalarios en donde la interdisciplina y la cooperación permiten a los gestores de enfermería proporcionar una práctica del cuidado de manera humanizada, integral y sin riesgos.
La dimensión del cuidado a las personas. Para Colliere M. F. ${ }^{23}$ cuidar es primero y ante todo, un acto de vida. Es decir, es el sentido que representa una importante variedad de actividades dirigidas a mantener y conservar la vida y permitir que ésta continúe y se reproduzca.

En tal caso, cuidar es un acto individual que uno se da a sí mismo, pero del mismo modo, es un acto de reciprocidad que se tiende a dar a cualquier persona que requiera temporal o definitivamente ayuda para asumir sus necesidades vitales. Para cuidar las enfermeras gestoras cuentan con tres aspectos básicos: los conocimientos aprendidos de diversas ciencias, la experticia se produce de la habilidad de sus cuidados individualizados y la aplicación de las tecnologías simples y complejas, garantizadas por la especialización de los aprendizajes. Por esta razón, los cuidados que la enfermería produce representan un conjunto de actividades que aseguran la continuidad de la vida y su reproducción. De acuerdo a la falta de salud de las personas, los cuidados son múltiples. Para ello, el personal de enfermería descubre y descifra quienes son las personas, sujetos de sus cuidados, para que con base en sus problemas de salud, se actúe con tecnologías apropiadas que gestionan y produzcan el bienestar.

Por último, el Modelo de gestión de enfermería del Hospital General "Dr. Manuel Gea González" toma en cuenta también a las teóricas de enfermería y sus escuelas. Por ejemplo, dice Amezcua M. ${ }^{24}$ la escuela de necesidades de Virginia Henderson y Dorothea Orem, de las interacciones de Imogene King, de los efectos deseables de Callista Roy y del cuidado transcultural de Madeleine Leininger, entre otras. Todas estas escuelas en el Modelo de gestión sirven de guía para la práctica clínica que acompañada de la práctica administrativa contribuye a reforzar el potencial y el cumplimiento de la identidad 
profesional de la enfermería en cualquier nivel de responsabilidad.

\section{CONCLUSIONES}

Los modelos de gestión del cuidado de enfermería son representaciones gráficas y abstractas de una realidad práctica en donde la persona es el sujeto de estudio de la enfermería y el objeto, es el cuidado que se brinda a las personas. De igual forma, el enfoque del modelo de gestión que se presenta está centrado en las dimensiones de administración, salud, enfermería y cuidados que en constante interacción permite movilizar los factores humanos y los recursos físicos, materiales y financieros para el bienestar de los individuos sanos o enfermos.

Resulta novedoso para la organización hospitalaria que la actual dirección de la Subdirección de Enfermería desarrolle un Modelo de gestión del cuidado de enfermería para mejorar los sistemas de organización con estrategias y coordinación de esfuerzos a fin de adecuar la planeación, organización y control de los procesos de atención y colaboración para garantizar la calidad de la atención de las personas.

Finalmente, es necesario señalar que este Modelo de gestión del cuidado nace por el esfuerzo del grupo líder de la Subdirección de Enfermería y con la confianza de que su aplicación práctica cuente con la participación de todo el personal de enfermería que facilite su comprensión y puesta en marcha, en beneficio de las personas y sus familias.

\section{AGRADECIMIENTO}

Se deja testimonio de reconocimiento a las jefes y supervisoras de enfermería por el entusiasmo mostrado en la construcción del Modelo de gestión del cuidado de enfermería que formó parte del Seminario de reingeniería de enfermería impartido en el Hospital General "Dr. Manuel Gea González”.

\section{CONFLICTO DE INTERESES}

Los autores declaran no tener Conflicto de Intereses en ésta publicación.

\section{REFERENCIAS}

1. Carvajal A. Teorías y modelos: formas de representación de la realidad. Instituto Tecnológico de Costa Rica. Escuela de Filosofía y Estudios Generales de la Universidad Tecnológica de Costa Rica. Costa Rica, 2018 p. 8 - 10. Disponible en: http:// revistas.tec.ac.cr/index.php/comunicacion/ article/view/1212/1118. Consultado 25 junio 2018.

2. Wartofsky M. Introducción a la filosofía de la ciencia. En Carvajal A. Teorías y modelos: formas de representación de la realidad. Comunicación. Instituto Tecnológico de Costa Rica. Escuela de Filosofía y Estudios Generales de la Universidad Tecnológica de Costa Rica. Costa Rica, 2018 p. 8 Disponible en: http://revistas.tec.ac.cr/index. $\mathrm{php} /$ comunicacion/article/view/1212/1118. Consultado 25 junio2018.

3. Martínez A. Desarrollo y definición de un Modelo de Gestión como paso previo a la innovación empresarial. Calidad. Enero-Marzo. México, 2018 p. 42 - 44. Disponible en: https://www.aec.es/c/document_ library/get_file?uud=9760bbb8-93ac-4de4-a201d5470864b3b3\&groupId=10128. Consultado 25 junio 2018. 
4. Pérez J, Merino M. Concepto de gestión: ¿ significado y qué es? Disponible en: http//:definición.de/ gestión. México, 2018 p.1- 2. Consultado 24 junio 2018.

5. Morti R. Gestión del cuidado de Enfermería. Cubana de Enfermería. Cuba, 2010: 26 (1): 1-2. Disponible en: http://www.bus.sld.cu/revistas/ enf/vol26110/enf01110.pdf. Consultado 20 junio 2018.

6. Kérouac Suzanne, Pepin Jacinthe, Ducharme Francine, Duquette André, Major Francine. El pensamiento enfermero. Ed. Elsevier- Masson. Madrid, 2007. p. 125-7.

7. Raile Alligood Martha, Marriner Tomey Ann. Modelos y teorías en enfermería. Ed. Elservier $8^{\mathrm{a}} \mathrm{ed}$. Madrid, 2015. p. 234-82.

8. Berbiglia VA, Banfield B. Teoría del déficit de autocuidado. En: Raile AM, Marriner TA. Modelos y teorías en enfermería. Ed. Elsevier. $8^{a}$ ed. Cap. 14. Madrid, 2018. p. 232-35.

9. Sieloff CL, Messmer PR. Marco de sistemas de interacción y teoría intermedia de la consecuencia de objetivos. En: Raile AM, Marriner TA. Modelos y teorías en enfermería. Ed. Elsevier. 8 ed. Cap. 15. Madrid, 2018. p. 293-7.

10. Phillips Kenneth D. Modelo de adaptación. En: Raile AM, Marriner TA. Modelos y teorías en enfermería. Ed. Elsevier. $8^{a}$ ed. Cap. 17. Madrid, 2018. p. 232-5.

11. Gunth MF. Seres humanos unitarios. En: Raile AM, Marriner TA. Modelos y teorías en enfermería. Ed.

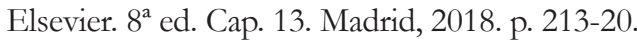

12. McFarland Marilyn R. Teoría de la diversidady de la universalidad de los cuidados culturales. En: Raile AM, Marriner TA. Modelos y teorías en enfermería. Ed. Elsevier. $8^{\text {a }}$ ed. Cap. 22. Madrid, 2018. p. 405-11.

13. Suasto Gómez WM. Diseño de la reingeniería de enfermería del Hospital General "Dr. Manuel Gea González”. Seminario permanente de reingeniería en enfermería. Hospital General "Dr. Manuel Gea González”. Ciudad de México. Agosto, 2018. p. 16.

14. Balseiro L. Principios de administración: En: Apoyo a los planes y programas de estudios de enfermería. Ed. Aztlán. 2a ed. México, 2010. p. 100.
15. Guilles DA. Gestión de enfermería: una explicación de los sistemas. Ed. Masson-salud. Madrid, 1994. p. 50-63.

16. Domínguez Ríos Víctor A, López Santillán Miguel A. Teoría general de sistemas, un enfoque práctico. Economía y Administración. Tecnociencia Chihuahua 2016;X(3):125-7. México. Disponible en: http://tecnociencia.vach.mx/numeros/ v10n3/Data/teoriageneraldesistemasunenfoque practico.pdf. Consultado: 27 junio 2018.

17. Tamayo A. Teoría general de sistemas. Ciencias Universidad Nacional de Colombia. Sede Manizales, Bogotá 1999:84-9. Disponible en: http://digital.unal.edu.co/57900/1/ teoriageneraldesistemas.pdf. Consultado: 27 junio 2018.

18. Torres M. Modelo de calidad de la atención media de Avedis Donabedian. Universidad Nacional Experimental Politécnica "Antonio José de Sucre". UNFXPO. Infocaster. Caracas, 2017;5(1):2. Disponible en: http://infocaster. blogspot.com/2011/10/modelo.de.la.calidad. de.la.atencion.media.html. Consultado: 27 junio 2018.

19. Donabedian A. Dimensión internacional de la evaluación y garantía de la calidad. Salud Pública de México. México, 1990; 32 (2): 7- 4.

20. Durán MM. Enfermería: desarrollo teórico e interdisciplinario. Facultad de Enfermería. Universidad Nacional de Colombia. Ed. INNOVAR. Bogotá, 1998. p. 77- 79.

21. González P. Guía de prácticas clínicas: herramienta educativa para la enseñanza de la metodología de la enfermera. Universidad de Madrid. Facultad de Enfermería, Fisioterapia y Podología. Madrid, 2018. p. 1-2.

22. Garley J. Algunas consideraciones metodológicas sobre el PAE como método científico de actuación profesional. Facultad de Ciencias Médicas. Departamento de Enfermería. Habana de Cuba, 2018. p. 8.

23. Colliere MF. Promover la vida. Ed.McGraw Hill Interamericana. México, 2009.p.211-12. Disponible en: http://www.facebook.com/ descargalibrosmedicina. Consultado: 25 junio 2018. 
Modelo de gestión del cuidado: innovación de enfermería para garantizar el bienestar... Suasto Gómez W. M., et al.

24. Amezcua M. Clasificaciones de los modelos de gestión y teóricas. Diapositivas. Facultad de Ciencias de la Salud. Universidad de Granada. Madrid, 2018. p.19. Disponible en: http://index-t.com/gomeres/ wp-contened/uploads/2014/12tema-10-escuelasde cuidado.pdf. Consultado: 29 junio 2018. 